\title{
Authentication of Damaged Hand Vein Patterns by Modularization
}

\author{
Chetana Hegde \\ RNSIT (Research Scholar at BU), \\ Bangalore, India \\ Email: chetanahegde@yahoo.co.in
}

\author{
Rahul Prabhu H, Sagar D S, Vishnu Prasad K, \\ P Deepa Shenoy and Venugopal K R \\ UVCE, Bangalore, India
}

\author{
L M Patnaik \\ Vice Chancellor, \\ DIAT, Pune, India
}

\begin{abstract}
As security is a major concern in present times, reliable authentication systems are in great demand. A biometric trait like the vascular pattern on the back of the hand of a person is unique and secure. A biometric system working on this principle often fails to authenticate a person either because of the varying hand posture or due to an injury altering the vein pattern. In this paper we propose an authentication system to overcome these disadvantages by modularizing the image and then comparing the features. This method of authentication reduces the False Rejection Ratio (FRR) and also False Acceptance Ratio (FAR) of the system.
\end{abstract}

\section{INTRODUCTION}

In the present times, security has become a critical issue in automated authentication systems. Biometrics is a science of identifying a person using their physiological or behavioral characteristics [1]. Biometric traits are difficult to counterfeit and hence results in higher accuracy when compared to other methods such as using passwords and ID cards. Human physiological and/or behavioral characteristic can be used as a biometric characteristic when it satisfies the requirements like Universality, Distinctiveness, Permanence and Collectability. However, in a practical biometric system, one needs to consider some other issues too like Performance, Acceptability and Circumvention [2]. Keeping all these requirements in mind, biometric traits like fingerprints, hand geometry, handwritten signatures, retinal patterns, facial images, etc. are used extensively in areas which require security access [2].

Vein pattern is one of the traits used in biometric security systems. Veins are the network of blood vessels underneath a persons skin. Since, the veins are hidden under the skin it is almost impossible for an imposter to copy the vein pattern. The properties mentioned earlier hold good for vein patterns. It is easily collectable with the proper devices such as a conventional Charge Coupled Devices (CCD) or an Infrared camera. Using vein authentication helps to create a balance between safety, economy, handiness, and social acceptance [3].

Vein pattern recognition is one of the most secure biometric traits. However, one of the major drawbacks of various systems proposed earlier is the inability to account for vein injuries and authenticate the person successfully. There will be some critical situations where the person has to be authenticated but is being rejected because of hand vein damage. This may happen particularly in the areas where injuries are common like defense, mines and high security research organizations.
For example consider a situation where a scientist has suffered injury to his hand in the course of carrying out an experiment. If this scientist wishes to enter another block of the organization, the security system may not authenticate him.

Such inefficiency can be overcome by various methods. For example, more than one biometric trait say, iris and fingerprint, of a person can be stored in the database and matched with the corresponding trait. This is the basic idea behind the bimodal biometric systems. But this increases the time spent at the security access system as each of the traits, here the iris and fingerprint, have to be scanned one at a time. This results in lesser efficiency with respect to time. In this paper we have developed a method for reducing the FRR to overcome the aforementioned drawback without compromising on FAR, efficiency and level of security offered by the system where a bimodal biometric authentication is involved.

This paper is organized as follows: Section II deals with the related work and Section III presents the architecture and model. Section IV is about problem definition. Section V describes the implementation of the proposed algorithm and the performance analysis. Section VI contains the conclusions.

\section{RELATED WORK}

A brief survey of the related work in the area of vein pattern recognition and authentication using vein pattern is presented in this section. Vein pattern recognition has been a field of keen interest among researchers since its dawn. Many methods have been put forward for identification and authentication. The initial step is to capture the hand vein image. The most commonly used technique is to capture the thermal image of back side of the hand to obtain the vein pattern.

Thermal images can be obtained in two ways, far-infrared (FIR) and near-infrared (NIR). Both methods have their own advantages and disadvantages. FIR thermography has difficulties in capturing the vein patterns of palm as it is sensitive to ambient conditions and human body conditions and does not provide a stable image quality. However, it is more suitable for capturing the large veins on the back of the hand. NIR produces good quality images when capturing the vein patterns from the back of the hand and the palm. Also, it is more tolerant to changes in environmental and body conditions. The main drawback of NIR thermography is that the image gets disrupted easily due to external skin features such as hair and 
line patterns. Therefore, for better results a device which uses NIR is chosen for capturing the images [1]. Using NIR lighting by CCD cameras, a verification system was developed and statistical performance measures were analyzed. This method also verified that every hand vein pattern is unique for each person and each hand [5].

At the intial stages of research on vein patterns, the vein structure information of the vein patterns was displayed on a VDU and plotted manually onto acetate [8], [9]. The image was traced to enable feature extraction and further analysis to take place away from the graphics display. Detection of vein structures was determined in most cases by viewing the IR images using the intensity and contrast controls on the VDU to provide for optimal detection of structure. This approach while possibly being useful for comparison of images is inadequate in the development of a practical automated system. Indeed the British Technology Group (BTG) researchers have since proposed representing each vein by a vector which has position and angle and can be regarded as a short straight line approximating a length of vein centreline [10]. They also found that a local contrast enhancement function and thresholding can be used to produce binary images of vein patterns from digital images of the back of hands. This is because, the binary images processed in this way are sufficiently consistent that it is feasible to employ a two dimensional geometric matching method to decide if a presented image is of an imposter [10].

But, automatically segment the vein patten from digital image and then to obtain a medial axis representation of the vein pattern is found to offer more faithful and robust representation of vein patterns [11]. This representation, in binary matrix format, can be considered as a vein signature which can uniquely identify an individual. The segmentation and skeletonization algorithms are based on mathematical morphology which provides an approach for processing digital images based on simplifying image data preserving their essential shape characteristics and eliminating irrelevancies [10]. But, mathematical morphology concerns the analysis of binary images by means of probing with structuring element [12]. The theory has been extended to grey-tone images and to complete lattices [13]. Structuring elements are geometric shapes which interact in what amounts to a fitting operation, with the image under investigation. In this way, geometric detail can be selectively removed from the image. Effectively, the image is reduced to a sort of caricature which is more expressive than the actual initial phenomenon [14].

Several algorithms have been developed to authenticate a person based on hand vein patterns. A hand vein authentication system using fast spatial correlation of hand vein patterns proposed by Mohamed Shahin et al., [4] uses correlation and rigid registration technique for authentication. Here, the matching percentage is calculated as the ratio of the count of overlapped white pixels between input images to the number of white pixels in one of the two input images. C. L. Lin et al., [7] proposes to extract feature points of the vein patterns (FPVPs) by modifying the basic tool of watershed transformation. Multiresolution representations of images with
FPVPs were obtained using multiple multiresolution filters that extract the dominant points by filtering miscellaneous features for each FPVP. A hierarchical integrating function was then applied to integrate multiple features and multiresolution representations. The former was integrated by an inter-to-intra personal variation ratio and the latter is integrated by a positive Boolean Function. This methodology was found to authenticate a person with FRR and FAR as $2.3 \%$.

These methods and such other works proposed by T. Tanaka et al., [6] etc. gives quite promising results in authenticating a person with acceptable FAR and FRR. But all these techniques fail when damage occurs for a hand vein. In this paper, we propose a technique of modularizing the hand vein image and then computing the features. Authentication is done by comparing module-wise features.

\section{ARCHITECTURE AND MODEling}

Having the database of hand-vein images of all the people in an organization consumes more space and the complexity of the system will increase. So, we suggest having an identification number (ID) for each person. The hand vein image features like number of veins, number of cross points and end points, positions of cross points and end points, and the number of modules the image occupies are stored. Also, we divide the image into modules and compute module-wise features. Thus, the database contains the ID and the corresponding image features and module features. During authentication, the person has to provide the ID and his hand vein is scanned. If the features of new image match with the corresponding features in the database, the person can be authenticated. Otherwise, the new image has to undergo modularization. Now, modulewise features are compared with corresponding module-wise features in the database. If the features match for specified number of modules, then also the person can be authenticated.

The architectural diagram for the proposed algorithm is shown in Fig. 1. The various steps involved are explained here under.

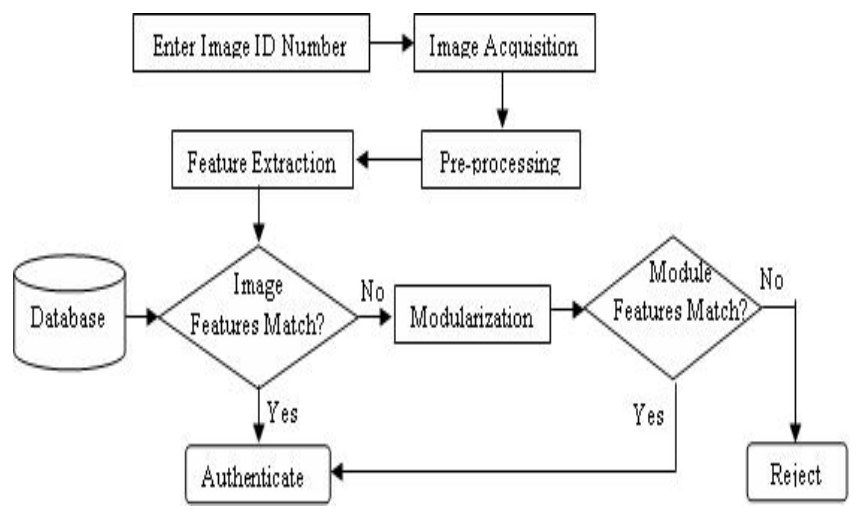

Fig. 1. Architectural Diagram

\section{A. Image acquisition}

A commercially available, low cost, monochrome CCD camera fitted with an IR filter is used to capture the thermal 
image of the hand. The filter helps in reducing the background noise. The image obtained is of lower quality but with reasonable clarity.

\section{B. Pre-processing}

The captured image is a gray-scale image. The region of interest is extracted by seperating background and foreground area of the image. Fig. 2(a) and Fig. 2(b) shows the original image and the region of interest.

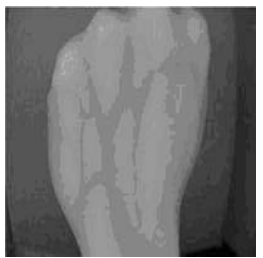

(a) Captured Image

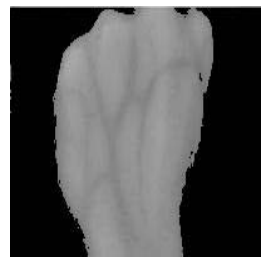

(b) Region of Interest
Fig. 2. Test Image1

The edges are detected by looking for local maxima of the gradient of image. The gradient is calculated using the derivative of a Gaussian filter. The method uses two thresholds, to detect strong and weak edges, and includes the weak edges in the output only if they are connected to strong edges. The outer edge of the hand is eliminated as we are interested only in vascular tree structure of hand veins. Then morphological filters are applied on this image to remove the noise and to increase the intensity of the image. Fig. 3(a) shows the image after edge detection. Morphological opening is applied to smoothen the contour of the edges and to eliminate thin protrusions. Then the image is dilated to expand the obtained edges. Fig. 3(b) shows the image after dilation.
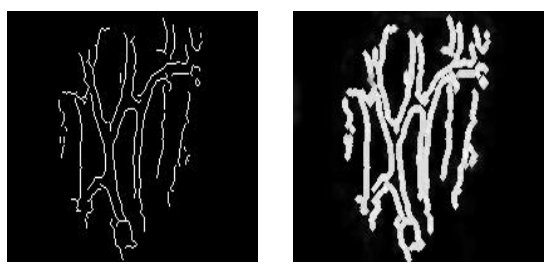

(a) Image after Detect- (b) Image after Dilation ing the Edge

Fig. 3. Edge Detection and Dilation for Test Image1

The dilated image contains holes in-between the vascular tree structure as shown in the Fig 3(b). These holes are filled based on set dilations, complementations and intersections. The procedure of region filling is done using the formula:

$$
X_{k}=\left(X_{k-1} \oplus B\right) \cap A^{c}, k=1,2,3, \ldots
$$

Here, $X_{0}=p$, and $B$ is the symmetric structuring element, $p$ being any arbitrary point inside the boundary. $A$ denotes a set containing a subset whose elements are 8-connected boundary points of a region. $A^{c}$ is the complement of the set $A$. The region filling algorithm terminates when $X_{k}=X_{k-1}$. The set
$X_{k} \cup A$ contains the filled set and its boundary. Fig. 4(a) shows the image after region filling.

Now, morphological thinning is applied to reduce the binary object or shape in an image to strokes that are single pixel wide. The thinning of an image $A$ by a structuring element $B$, denoted $A \otimes B$, can be defined in terms of the hit-or-miss transform as:

$$
A \otimes B=A-(A \odot B)=A \cup(A \odot B)^{c}
$$

This method of thinning will remove the pixels so that an object without holes shrinks to a minimally connected stroke and an object with holes shrinks to a ring halfway between the hole and outer boundary. Fig. 4(b) shows the image after morphological thinning.

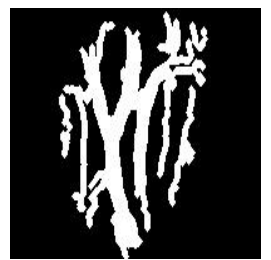

(a) Image after Region (b) Image after MorphoFilling

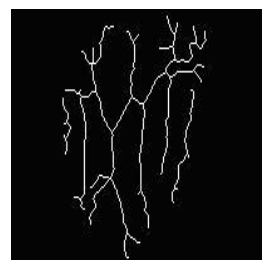
logical Thinning
Fig. 4. Region Filling and Thinning for Test Image1

After this step, we use clipping technique to align the tree structure at top-left corner. Clipping is done using spatial coordinates of the image. This alignment is required to overcome the anomalies arising due to the movement of hand during image acquisition. Fig. 5(a) shows the image after clipping.

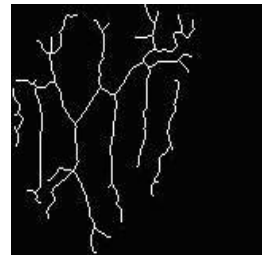

(a) Image after Clipping (b) Image after modularization
Fig. 5. Clipping and Modularization for Test Image1

\section{Feature Extraction}

The features extracted must be non-trivial and must not vary for a given person over time. Two kinds of features are extracted viz. image features, which are the features of image as a whole, and the module features that are features pertaining to a particular module. Image features include number of veins in the hand, number of cross points and end points, position of cross points and end points and number of modules the image occupies. Module features include number of veins in the module, number of cross points and end points in the module and position of those cross points and end points in that module. 
Initially, we have to count number of veins. In practical implementations, the pixels in an image seldom characterize an edge completely because of noise, breaks in the edge from nonuniform illumination and other effects that introduce spurious intensity discontinuities. Thus, we need to use linking procedures to assemble edge pixels into meaningful edges. One approach that can be used to find and link line segments in an image is the Hough Transform. For detecting lines, Hough Transform uses the polar co-ordinates $r$ and $\theta$, where $r$ is the distance from the origin to the line along a vector perpendicular to that line and $\theta$ is the angle between the $x$-axis and this vector. Using this parameterization, the equation of the line can be written as:

$$
y=\left(-\frac{\cos \theta}{\sin \theta}\right) x+\left(\frac{r}{\sin \theta}\right)
$$

which can be rearranged to $r=x \cos \theta+y \sin \theta$. It is therefore possible to associate to each line of the image, an ordered pair $(r, \theta)$ which is unique if $\theta \in[0, \pi]$ and $r \in R$, or if $\theta \in[0,2 \pi]$ and $r \geq 0$.

Using Hough Transform, we obtain a vector containing radii of detected lines and a vector containing angles of detected lines for a given image. The number of radii present in the first vector gives the number of veins present in the image.

The Number of cross points and end points and their positions are determined by the Chord-to-Point Distance Accumulation (CPDA) Technique.

\section{Modularization}

The image is divided into 16 modules of equal size. Given an image, we will crop it using spatial co-ordinates, which is a rectangle of certain width and height. The output module may vary by one or two pixels from the size specified. This is because the output module includes all pixels in the input that are completely or partially encolsed by the rectangle. Fig. 5(b) shows the image after modularization. This figure contains all the 16 modules obtained. For each module, the features are extracted using Hough Transform and CPDA technique.

\section{E. Authentication}

This process involves a series of steps to authenticate a person.

1) The person who undergoes the authentication process should first enter the ID and get the hand scanned with the scanning device.

2) The hand vein image captured undergoes pre-processing and feature extraction to extract the features.

3) The extracted features are verified against the features retrieved from the database stored for that ID.

4) Now, the percentage match between the several features of captured image and the corresponding features in the database is calculated using the formulae:

$$
P_{1}=\frac{a b s(N V C-N V D)}{N V D} \times 100
$$

$$
\begin{aligned}
& P_{2}=\frac{a b s(N C P C-N C P D)}{N C P D} \times 100 \\
& P_{3}=\frac{a b s(N E P C-N E P D)}{N E P D} \times 100
\end{aligned}
$$

Here, NVC and NVD denotes the number of veins in the captured image and in the database respectively. NCPC and NCPD represents the number of cross-points for the captured image and in the database respectively. NEPC and NEPD are the number of end points in the captured image and in the database respectively.

If all the values of $P_{1}, P_{2}$ and $P_{3}$ are greater than $90 \%$, then the person can be authenticated. Otherwise, that person will be rejected.

5) If a person has suffered hand injury, due to which the vein is damaged, the person may not be authenticated as there are possibilities of percentage match not crossing $90 \%$. In such cases the image captured is modularized and module features of each module are verified against the corresponding module features stored in the database.

6) Now for each module, the percentage match is calculated using the formulae stated above. If all the values of $P_{1}$, $P_{2}$ and $P_{3}$ for atleast 13 modules are greater than $90 \%$, then the person can be authenticated. Otherwise he will be rejected.

For the original image given in Fig. 2(a), the damaged image with minor injuries is given in Fig. 6. This image is modularized and features are extracted. We found that there is a feature-match for 15 modules and hence the person can be authenticated.

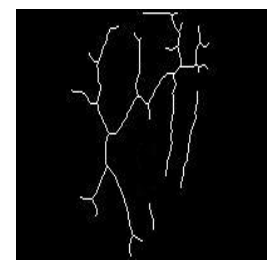

Fig. 6. Hand Vein Pattern when Test Image1 was Damaged

\section{Algorithm}

\section{A. Problem Definition}

Given an image in the format of GIF or JPEG $(150 \times 200)$, the objectives are:

(1) To compute image features like number of veins, number and positions of cross points and end points, number of modules that it may occupy.

(2) To divide the image into module and to compute the above mentioned features for each module and

(3) To compare image features as a whole or, if needed, to compare module-wise features for authenticating a person.

The basic assumption made here is that hand vein images are grey scale images. 


\section{B. Algorithm}

Four major functions are involved in the proposed technique. The first function is for edge detection and preprocessing. The algorithm for detecting the edge is given in Table I. The second function is used to compute number of veins in an input image using Hough Transform. The input image may be the entire image or it may be individual module after modularization. The algorithm for finding number of veins using Hough Transform is shown in Table II. The third function is for computing number of cross points and end points in a vein pattern using CPDA technique. The algorithm for CPDA is summarized in Table III. The last function is used for generating modules from a given image.

TABLE I

Edge Detection Algorithm

(1) The input image is smoothed using a Gaussian filter with a specified standard deviation $\sigma$, to reduce noise.

(2) The local gradient, $g(x, y)=\left[G_{x}^{2}+G_{y}^{2}\right]^{\frac{1}{2}}$, and edge direction, $\alpha(x, y)=\tan ^{-1}\left(\frac{G_{x}}{G_{y}}\right)$ are computed at each point. Here, $G_{x}$ and $G_{y}$ are first derivatives. An edge point is defined to be a point whose strength is locally maximum in the direction of the gradient.

(3) The edge points determined in Step 2 give rise to ridges in the gradient magnitude image. The algorithm then tracks along the top of these ridges and sets to zero all pixels that are not actually on the ridge top so as to give a thin line in the output, a process known as nonmaximal suppression. The ridge pixels are then thresholded using two thresholds $T 1$ and $T 2$, with $T 1<T 2$. Ridge pixels with values greater than $T 2$ are said to be strong edge pixels. Ridge pixels with values between $T 1$ and $T 2$ are said to be weak edge pixels.

(4) Finally, the algorithm performs edge linking by incorporating the weak pixels with that are 8-connected to the strong pixels.

TABLE II

Counting Number of Veins using Hough Transform

(1) Rotate the image pixel locations by $90^{\circ}-\theta$ so that they lie approximately along a vertical line.

(2) Sort the pixel locations by their rotated $x$-values.

(3) Locate the gaps by finding the differences between pixel values. Ignore small gaps; this has the effect of merging adjacent line segments that are separated by a small space.

(4) Return information about line segments that are longer than some minimum length threshold.

\section{Implementation And Performance Analysis}

The implementation of proposed technique is developed using MatLab 7.5. It is observed through simulation that creation of 16 modules for each image is optimum. We found that creating 9 modules will increase FRR. If we create 25 modules then there is a chance of cross-points falling at
TABLE III

Chord-to-Point Distance Accumulation Technique

(1) Input a binary image containing edge-map of hand vein.

(2) Extract the edge contours from the edge-map; fill the gaps in the contours.

(3) Compute curvature at a low scale for each contour to retain all true corners. All of the curvature local maxima are considered as initial corner candidates.

(4) Initial corner candidates are compared using an adaptive local threshold to remove the round corners.

(5) The angles of corner candidates are evaluated to eliminate any false corners due to quantization noise and trivial details. The above evaluation is based on a dynamic region of support, which changes according to its adjacent corner candidates.

(6) End points of contours are considered with an additional criterion.

the boundaries. Hence, we have constructed 16 modules for every image. The proposed algorithm is tested on hand vein images of several people for both left and right hands. The conventional methods are observed to reject images in which veins are damaged, and hence increasing FRR. But simulation results of our algorithm show that for minor injured hand veins, the authentication is possible. Thus FRR will be reduced. When the damage is severe, secure authentication is critical. So, we confine our algorithm to authenticate a person only if the damage is limited to three modules. If module features do not match for more than three modules, we reject the person. This approach is helping us not to increase FAR.

The simulation results on some images of both left hand and right hand are shown in Fig. 7 to Fig. 9. Fig. 7(a), 8(a) and 9(a) shows the original images before damage. Fig. 7(b), 8(b) and 9(b) shows the vascular tree structure of the hand vein before damage. The respective damaged hand vein images are shown in Fig. 7(c), 8(c) and 9(c). For the damaged image shown in Fig. 7(c), the damage was found in three modules and features in other modules match with the features of original image. So, we authenticate that person. For the damaged image shown in Fig. 8(c), the damage was found to be in five modules and hence there will be mis-match of features in five modules when compared with original image. Thus, we reject the person. Fig. 9(c) shows the image with minimum damage, only in one module. Since there is feature-match in all other 15 modules, we accept the person.

The conventional methods for authentication using hand vein patterns [4], [6], [7] were found to have FAR and FRR about 2.3\%. The proposed technique on modularization resulted in FAR of about $2.1 \%$ and FRR about $1.274 \%$. Thus, modularization forces reasonable reduction in FRR.

\section{CONCLUSIONS}

In this paper, we propose an efficient way for authentication using hand vein patterns, when damage has occurred to vein patterns. The proposed technique uses modularization of hand vein images. The method developed here has a greater efficiency with respect to time required for authentication, 


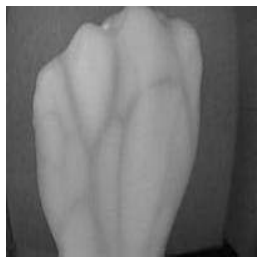

(a) Original Image

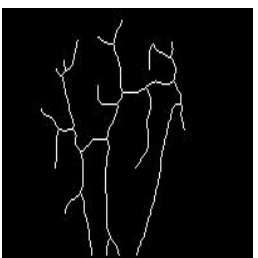

(b) Vascular Tree Structures Before Damage

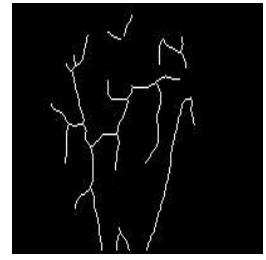

(c) Image Showing Damaged Vein Pattern

Fig. 7. Test Image2

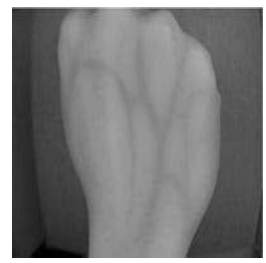

(a) Original Image
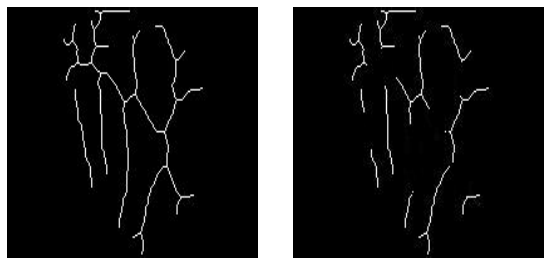

(b) Vascular Tree Struc- (c) Image Showing tures Before Damage Damaged Vein Pattern

Fig. 8. Test Image3

compared to a similar multimodal authentication system. And also it reduces FAR and FRR. For every initial rejection, the image will undergo modularization irrespective of vein damage. That is, even when the person is not genuine the result is known only after modularization. As a future work, we will overcome this issue by introducing some more hand-vein features like shape of vein curves. Also, the authentication for a rotated hand-vein image also can be taken as a future work.

\section{REFERENCES}

[1] W. Lingyu and G. Leedham, "Near and Far Infrared Imaging for Vein Patter Biometrics," Proc. of IEEE Int. Conf. on Video and Signal Based Surveillance (AVSS 06), Nov. 2006, pp. 52-52.

[2] A K Jain, A Ross and S Prabhakar, "An Introduction to Biometric Recognition," IEEE Trans. on Circuits Sys, vol. 14, no. 1, Jan. 2004, pp. 4-20.

[3] S. Watanabe, T. Tanaka, E. Iwata, "Biometric Authentication using Phase Only Correlation with Compensation Algorithm for Rotation," SICEICASE International Joint Conference, 2006, pp. 371-3715.

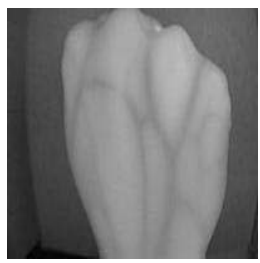

(a) Original Image

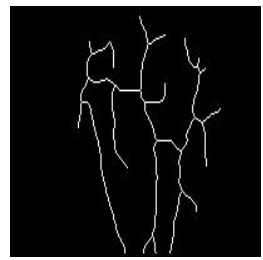

(b) Vascular Tree Structures Before Damage

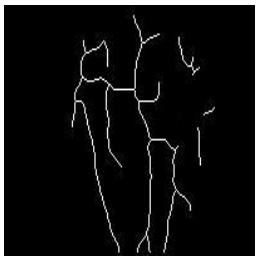

(c) Image Showing Damaged Vein Pattern

Fig. 9. Test Image4

[4] M. Shahin, A. Badawi and M. Kamel, "Biometric Authentication Using Fast Correlation of Near Infrared Hand Vein Patterns," Int. Jour. of Biomedical Sciences, vol. 2, no. 3, April 2007, pp. 141-148.

[5] A. M. Badawi, "Hand Vein Biometric Verification Prototype: A Testing Performance and Patterns Similarity," Proc. of Int. Conf. on Image Processing, Computer Vision, and Pattern Recognition, 2006, pp. 3-9.

[6] T. Tanaka and N. Kubo, "Biometric Authentication by Hand Vein Patterns," SICE, Annual Conference in Sapporo, Aug. 2004, pp. 249-253.

[7] C. L. Lin and K. C. Fan, "Biometric Verification using Thermal Images of Palm-Dorsa Vein Patterns," IEEE Trans. on Circuits Sys Video Tech., vol. 14, no. 2, Feb. 2004, pp. 199-213.

[8] P. MacGregor and R. Welford, "Veincheck: Imaging for Security and Personnel Identification," Advanced Imaging, vol. 6, no. 7, 1991, pp. 52-56.

[9] P. MacGregor and R. Welford, "Veincheck Lends a Hand for High Security," Sensor Review, vol. 12, no. 3, 1992, pp. 19-23.

[10] P. L Hawkes and D. O. Clayden, "Veincheck Research for Automatic Identification of People," prsented at the Hand and Fingerprint Seminar at NPL., Sept. 1992.

[11] J. M. Cross and C. L. Smith, "Thermographic Imaging of the Subcutaneous Vascular Network of the Back of the Hand for Biometric Identification," Proc. of IEEE 29th Annual International Carnahan Conference, 1995 , pp. 20-35.

[12] E. R. Dougherty, (Ed.)., in Mathematical Morphology in Image Processing, New York: Marcel Dekker, Inc., 1993.

[13] D. Lake, "How can I see What I'm Looking at?," Lighting and Image Accuracy, Quality. Advanced Imaging, vol. 9, no. 5, 1994, pp. 34-38.

[14] W. Kuczborski, Y. Attikiouzel and G. Crebbin, "Video Rate Morphological Processor based on a Redundant Number Representation," In B. G. Batchelor, M. J. Chen F. W. Waltz (Eds.), Machine Vision, Architectures, Integration and Applications, Bellingham:SPIE, 1994, pp. 249-260. 